\title{
Symmetry-based approach to electron-phonon interactions in graphene
}

\author{
J. L. Mañes \\ Departamento de Física de la Materia Condensada \\ Universidad del País Vasco, Apdo. 644, E-48080 Bilbao, Spain
}

(Dated: February 4, 2008)

\begin{abstract}
We use the symmetries of monolayer graphene to write a set of constraints that must be satisfied by any electron-phonon interaction hamiltonian. The explicit solution as a series expansion in the momenta gives the most general, model-independent couplings between electrons and long wavelength acoustic and optical phonons. As an application, the possibility of describing elastic strains in terms of effective electromagnetic fields is considered in detail, with an emphasis on group theory conditions and the role of time reversal symmetry.

PACS numbers: 63.20.Kr, 63.22.+m, 71.15.Rf
\end{abstract}

\section{INTRODUCTION}

Symmetry plays an obiquitous role in solid state physics, where invariance of the system under symmetry transformations imposes strong constraints on the form of hamiltonians and other physical observables $\frac{1.2}{.2}$ In computations of phonon spectra, the choice of convenient symmetry-adapted coordinates simplifies the diagonalization of the dynamical matrix ${ }^{\frac{3}{3}}$ Instead of a $3 N \times 3 N$ matrix, where $N$ is the number of atoms in the unit cell, we end up with a collection of smaller matrices, one per inequivalent IR (irreducible representation), with a dimension equal to the multiplicity of that representation in the 'mechanical' representation. Similar simplifications take place in the computation of electronic bands.

An important caveat is that the symmetry which is effective in simplifying the problem is not the whole space group of the crystal, but the little group 1 that preserves the wavevector of the electrons or phonons. $\stackrel{4}{=}$ Thus, symmetry-based methods are really powerful around points and lines of high symmetry in the Brillouin zone. Since electron-phonon interactions involve generic wavevectors with trivial little groups, it would seem that symmetry considerations can not be very helpful in that case. Indeed, many computations of electronphonon interactions are based on the use of specific models (electron-ion interactions,$\frac{5.6}{,}$ modulated hoppings,,$\frac{7.8 .9}{6 .}$

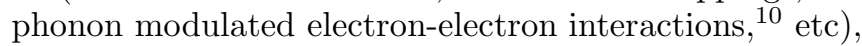
and little or no use is made of the symmetries of the system.

On the other hand, long-wavelength phonons play a crucial role in many physical processes of interest, such as transport phenomena, where electron-phonon interactions are essential. ${ }^{5.6}$ The elements of the dynamical matrix for long wavelength phonons (acoustic and optical) can be expanded as a power series in the phonon wavevector $q$, and this expansion is strongly constrained by the little group of the $\Gamma$ point, which of course coincides with the space group of the crystal. Unfortunately, low energy electrons lie near the Fermi surface, where typical points lack any symmetry.

Graphene $\frac{11,12}{12}$ is a remarkable exception in this regard. Instead of an ordinary Fermi surface, undopped monolayer graphene has two isolated, high symmetry Fermi points. Thus, as long as we restrict ourselves to long wavelength phonons and low energy electrons, which are necesarily close to the Fermi points, the interaction hamiltonian will be strongly constrained by symmetry. In fact, in Section 4 we will be able to write the most general interaction hamiltonian as an explicit series expansion around the $\Gamma$ point (for phonon wavevectors) and the Fermi points (for electron wavevectors).

One possible application of the hamiltonian obtained here is to many-body computations,,$\frac{6,13,14}{}$ where analytic expressions are often preferred over numerically defined functions. In the usual approach a concrete model, such as one based on phonon-modulated nearest neighbors hoppings, is used to compute an interaction hamiltonian which later is expanded to the required order in the momenta $.0,13,15$ Alternatively, one can take the interaction hamiltonian given in Section 4 as a power expansion, and keep terms up to the order desired. Obviously, less effort is involved by this method, and one is sure that all possible couplings have been included, whereas a particular model may miss some of then. The obvious drawback is that symmetry arguments by themselves do not give a clue as to the values of the parameters. Another, very different application of the electron-phonon hamiltonian is presented in Section 5, where we investigate a description of elastic strains in terms of effective electromagnetic fields $\frac{16}{6}$

This paper is organized as follows. In Section 2 we review the symmetries of monolayer graphene and the transformation properties of electrons and phonons. Section 3 presents a study of the dynamical matrix including the form of the long wavelength normal modes. The phonon-electron interactions are computed in Section 4, and the results are used in Section 5 to study the possibility of interpreting trilinear electron-phonon couplings in terms of effective scalar and vector potentials. The conclusions are presented in Section 6. 


\section{SYMMETRIES}

We begin this section with a description of electrons and phonons in monolayer graphene, including the choice of the symmetry-adapted modes to be used in the rest of this paper. We then proceed to study their transformation properties.

\section{A. Electrons and phonons}

Monolayer graphene consists of a honeycomb lattice. The corresponding hexagonal Bravais lattice is generated by $\vec{t}_{1}=(a \sqrt{3}, 0)$ and $\vec{t}_{2}=(a \sqrt{3} / 2,3 a / 2)$, where $a$ is the distance between nearest neighbours. (See Fig. 1). The unit cell contains two atoms, with positions given by $\vec{r}_{1}=\vec{t}_{1} / 3+\vec{t}_{2} / 3$ and $\vec{r}_{2}=2 \vec{r}_{1}$. The reciprocal lattice is generated by $\vec{g}_{1}=(2 \pi / \sqrt{3} a,-2 \pi / 3 a)$ and $\vec{g}_{2}=(0,4 \pi / 3 a)$, with $\vec{t}_{i} \cdot \vec{g}_{j}=2 \pi \delta_{i j}$. Corresponding to the two atoms in the unit cell, we may define two Bloch wave functions

$$
\Phi_{i}(\vec{K})=\sum_{\vec{t}} e^{i \vec{K} \cdot\left(\vec{r}_{i}+\vec{t}\right)} \Phi\left(\vec{r}-\vec{r}_{i}-\vec{t}\right) \quad, \quad i=1,2
$$

where the sum runs over all the points in the direct lattice, i.e., $\vec{t}=n_{1} \vec{t}_{1}+n_{2} \vec{t}_{2}$ and $\Phi(\vec{r})$ is a real $\pi$-type atomic orbital. As is well known, a simple tight-binding computation ${ }^{17,18}$ yields a spectrum with two Fermi points located at $\vec{K}_{1}=-2 \vec{g}_{1} / 3-\vec{g}_{2} / 3$ and $\vec{K}_{2}=-\vec{K}_{1}$. Near the two Fermi points, the hamiltonian can be linearized and one finds

$$
H\left(\vec{K}_{1}+\vec{k}\right) \sim \frac{3}{2} a t\left(\begin{array}{cc}
0 & k^{*} \\
k & 0
\end{array}\right), k \equiv k_{x}+i k_{y}
$$

where $t$ is the hopping integral, and $H\left(-\vec{K}_{1}+\vec{k}\right)=$ $H^{*}\left(\vec{K}_{1}-\vec{k}\right)$. Thus, the low energy electronic excitations behave like massless Dirac fermions 19,20 with relativistic spectrum $E= \pm v_{F}|k|$. Henceforth we assume our units are such that $v_{F}=3 a t / 2=1$.

In this paper we want to consider the interaction between electrons near the Fermi points $\left\{\vec{K}_{1},-\vec{K}_{1}\right\}$ and phonons $Q(\vec{q})$ for small values of $q$. Due to invariance under reflection by the horizontal plane $\sigma_{h}$, only in-plane modes can couple linearly to electrons. Thus, for most of this paper we consider only in-plane modes, which are specified by giving the horizontal displacements $u(\vec{t})=\left(x_{1}, y_{1}, x_{2}, y_{2}\right)$ of the two atoms in the unit cell with origin at the lattice point $\vec{t}$. See however Section 5, where quadratic couplings of out-of-plane acoustic modes are considered in the long wavelength limit.

We can choose a basis of symmetry-adapted modes with particularly simple transformation properties by noting that, under a point group transformation, a mode undergoes two changes: The vectors describing atomic displacements are rotated or reflected according to the vector representation, and there is a permutation among
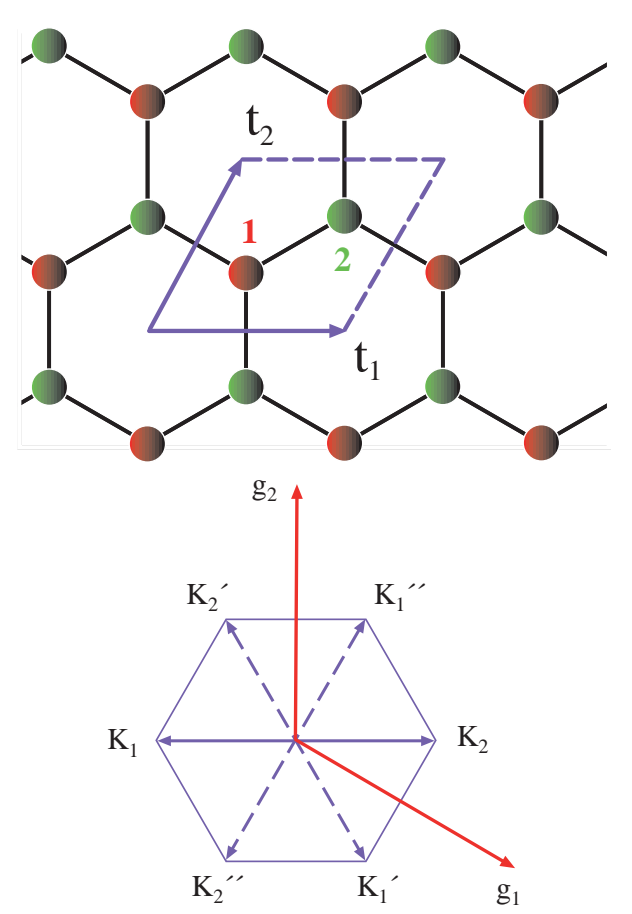

FIG. 1: (Color online) Top: Direct lattice and unit cell for monolayer graphene. Bottom: First Brillouin zone and Fermi points. The vectors $\vec{K}_{1}^{\prime}, \vec{K}_{1}^{\prime \prime}\left(\vec{K}_{2}^{\prime}, \vec{K}_{2}^{\prime \prime}\right)$ are equivalent to $\vec{K}_{1}$ $\left(\vec{K}_{2}\right)$.

equivalent atoms. This last effect may give rise to annoying phases in the transformation matrices, which can be avoided by incorporating appropriate phases into the definitions of the modes. The use of circular rather that linearly polarized modes simplifies the effect of rotations.

Our choice of in-plane modes is given in Table【. In the long wavelength limit $q a<<1$, the phases vanish $e_{i}(q) \rightarrow$ 1 and the unprimed (primed) modes become circularly polarized acoustic (optical) normal modes respectively (see Section 3 for details).

\begin{tabular}{c|cccc} 
& $u_{1}$ & $u_{2}$ & $u_{1}^{\prime}$ & $u_{2}^{\prime}$ \\
\hline \hline$x_{1}$ & $e_{1}(q)$ & $e_{1}(q)$ & $e_{1}(q)$ & $e_{1}(q)$ \\
$y_{1}$ & $i e_{1}(q)$ & $-i e_{1}(q)$ & $i e_{1}(q)$ & $-i e_{1}(q)$ \\
$x_{2}$ & $e_{2}(q)$ & $e_{2}(q)$ & $-e_{2}(q)$ & $-e_{2}(q)$ \\
$y_{2}$ & $i e_{2}(q)$ & $-i e_{2}(q)$ & $-i e_{2}(q)$ & $i e_{2}(q)$
\end{tabular}

TABLE I: In-plane symmetry-adapted modes for monolayer graphene. Here $e_{i}(q)=\frac{1}{2} e^{i \vec{q} \cdot \vec{r}_{i}}$, where $\left\{\vec{r}_{i}\right\}$ are the equilibrium positions of the two atoms in the unit cell.

This basis is used to expand general in-plane displacements $u(\vec{t})=\left(x_{1}, y_{1}, x_{2}, y_{2}\right)$ as follows

$$
u(\vec{t})=\sum_{q}\left(\sum_{i=1}^{2} Q_{i}(\vec{q}) u_{i}(\vec{q})+\sum_{i=1}^{2} Q_{i}^{\prime}(\vec{q}) u_{i}^{\prime}(\vec{q})\right) e^{i \vec{q} \cdot \vec{t}}
$$




\section{B. Transformation properties}

The space group of monolayer graphene is symmorphic, with point group $D_{6 h}$ (see ref. ${ }^{2}$ for conventions and notation). $D_{6 h}$ is generated by $\left\{C_{6}^{+}, \sigma_{v 1}, \sigma_{h}\right\}$, where $C_{6}^{+}$ is a counterclockwise rotation by $\pi / 3$ around the $z$ axis, $\sigma_{v 1}$ is a reflection by the $x z$ plane and $\sigma_{h}$ is a reflection by the horizontal $x y$ plane that contains the graphene layer. However, given the planar structure of the system and as long as we are restricted to in-plane modes, we will consider instead the normal subgroup $C_{6 v}$, generated by $\left\{C_{6}^{+}, \sigma_{v 1}\right\}$. The little point group of $\vec{K}_{1}$ is $\hat{G}_{\vec{K}_{1}}=C_{3 v}$, generated by $\left\{C_{3}^{+}, \sigma_{v 1}\right\}$. The star of $\vec{K}_{1}$ consists of the two Fermi points $\left\{\vec{K}_{1},-\vec{K}_{1}\right\}$. Since the binary rotation $C_{2}$ around the $z$ axis transforms $\vec{K}_{1}$ into $-\vec{K}_{1}$, it is convenient to use $\left\{C_{3}^{+}, \sigma_{v 1}, C_{2}\right\}$ as a (redundant) set of generators for $C_{6 v}$.

The action of the generators of $C_{6 v}$ on the modes in Table $\mathrm{I}$ is given in Appendix A. As a result of a symmetry operation $g$, a displacement with wavevector $q$ is transformed into a new displacement with wavevector $g q$ and coordinates $\left\{g Q_{i}, q Q_{i}^{\prime}\right\}$. Defining $Q \equiv\left\{Q_{1}, Q_{2}\right\}$ and $Q^{\prime} \equiv\left\{Q_{1}^{\prime}, Q_{2}^{\prime}\right\}$, the transformation properties can be written

$$
g Q(g q)=T(g) Q(q) \quad, \quad g Q^{\prime}(g q)=T^{\prime}(g) Q^{\prime}(q)
$$

where $T^{\prime}\left(C_{2}\right)=-T\left(C_{2}\right)=\mathbf{1}$ is the unit matrix, and

$$
\begin{gathered}
T\left(\sigma_{v 1}\right)=-T^{\prime}\left(\sigma_{v 1}\right)=\sigma_{x}=\left(\begin{array}{cc}
0 & 1 \\
1 & 0
\end{array}\right) \\
T\left(C_{3}^{+}\right)=T^{\prime}\left(C_{3}^{+}\right)=\Omega \equiv\left(\begin{array}{cc}
\omega^{*} & 0 \\
0 & \omega
\end{array}\right)
\end{gathered}
$$

with $\omega \equiv \exp (2 \pi i / 3)$.

Another important symmetry is time reversal, which acts by complex conjugation. According to Table I,

$$
\mathcal{T}: u_{1}^{*}(q)=u_{2}(-q) \quad, \quad u_{2}^{*}(q)=u_{1}(-q)
$$

with identical action on $u_{i}^{\prime}$. Then, reality of a general displacement (3) implies

$$
Q^{*}(q)=\sigma_{x} Q(-q) \quad, \quad Q^{\prime *}(q)=\sigma_{x} Q^{\prime}(-q)
$$

We now turn our attention to the electrons. Since low energy electronic excitations are located near the two Fermi points, it is convenient to change variables, $\vec{K}= \pm \vec{K}_{1}+\vec{k}$. Correspondingly, we define Bloch functions $\Phi_{i \pm}(k)$ in the neighbourhoods of the Fermi points

$$
\Phi_{i \pm}(k) \equiv \Phi_{i}\left( \pm \vec{K}_{1}+\vec{k}\right)
$$

The action of the generators of $C_{6 v}$ on the wavefunctions is given in Appendix A. As long as we do not consider the spin, time reversal acts simply by complex conjugation. According to (1), $\Phi_{i}^{*}(\vec{K})=\Phi_{i}(-\vec{K})$. Thus time reversal exchanges the two Fermi points and we have

$$
\begin{aligned}
\mathcal{T}: & \Phi_{i+}(k) \rightarrow \Phi_{i+}^{*}(k)=\Phi_{i-}(-k) \\
& \Phi_{i-}(k) \rightarrow \Phi_{i-}^{*}(k)=\Phi_{i+}(-k)
\end{aligned}
$$

As both time reversal $\mathcal{T}$ and the binary rotation $C_{2}$ around the $z$ axis reverse the sign of the wavevector, the combined symmetry $\mathcal{T} C_{2}$ preserves the Fermi points. Note however, that the two atoms are exchanged

$$
\mathcal{T} C_{2}: \Phi_{1+}(k) \leftrightarrow \Phi_{2+}(k), \Phi_{2-}(k) \leftrightarrow \Phi_{1-}(k)
$$

This combined symmetry plays an important role in the computation of electron-phonon interactions in Section 4 and is behind the topological stability of Fermi points in mono and multi-layer graphene. ${ }^{22}$

\section{DYNAMICAL MATRIX}

The modes in Table I have simple transformation properties, but they do not correspond to normal modes with well defined frequencies. In general, these must be obtained by numerical diagonalization of the dynamical matrix. $23,24,25$ However, in the long wavelength regime one can easily obtain useful analytical results by exploiting the symmetries of the system.

The symmetries constrain the form of the dynamical matrix $V(q)$ defined by

$$
E_{p}=\frac{m}{2} \sum_{q} \mathbf{Q}^{\dagger}(q) V(q) \mathbf{Q}(q)
$$

where $\mathbf{Q}=\left\{Q, Q^{\prime}\right\}$ stands for the four symmetryadapted coordinates in (3), $m$ is the mass of the carbon atom and the sum is over the first Brillouin zone. The hermitian matrix $V$ can be decomposed into $2 \times 2$ blocks

$$
V(q)=\left(\begin{array}{cc}
D & M \\
M^{\dagger} & D^{\prime}
\end{array}\right)
$$

associated to the unprimed and primed modes. Then, invariance under a spatial symmetry (4) gives

$$
\mathbf{T}(g) V(q) \mathbf{T}^{\dagger}(g)=V(g q)
$$

with

$$
\mathbf{T}(g)=\left(\begin{array}{cc}
T(g) & 0 \\
0 & T^{\prime}(g)
\end{array}\right)
$$

whereas invariance under time reversal (7) implies

$$
\mathcal{T} V^{*}(q) \mathcal{T}=V(-q), \quad \mathcal{T}=\left(\begin{array}{cc}
\sigma_{x} & 0 \\
0 & \sigma_{x}
\end{array}\right)
$$

Neglecting initially the acoustic-optical mixing due to $M$, we can obtain an approximation to acoustic frequencies and modes by solving a $2 \times 2$ eigenvalue problem

$$
D(q) u(q)=\omega^{2}(q) u(q)
$$

where $u(q)$ is a linear combination of the two unprimed modes given in table I. As shown in Appendix B, the 
symmetry constrains the form of the hermitian matrix $D$ to

$$
D(q)=\left(\begin{array}{cc}
a(q) & b(q) \\
b^{*}(q) & a(q)
\end{array}\right)
$$

where $a(q)$ and $b(q)$ are even functions of $q$ that satisfy

$$
\begin{gathered}
a(\omega q)=a(q), b(\omega q)=\omega b(q) \\
a\left(q^{*}\right)=a(q), \quad b\left(q^{*}\right)=b^{*}(q)
\end{gathered}
$$

The eigenvalue problem is solved by

$$
\omega_{ \pm}^{2}(q)=a(q) \pm|b(q)|
$$

with normal modes given by

$$
u_{ \pm}(q)=\frac{1}{\sqrt{2}}\left(u_{1}(q) \frac{b(q)}{|b(q)|} \pm u_{2}(q)\right)
$$

In the absence of acoustic-optical interactions, this is the most general acoustic spectrum compatible with the symmetries of the system. For small $q$ this solution admits a simple geometric interpretation. Expanding $a(q)$ and $b(q)$

$$
\begin{aligned}
& a(q)=a_{2}|q|^{2}+a_{4}|q|^{4}+O\left(q^{6}\right) \\
& b(q)=b_{2} q^{* 2}+b_{4}|q|^{2} q^{* 2}+\tilde{b}_{4} q^{4}+O\left(q^{6}\right)
\end{aligned}
$$

where all the constants are real, and keeping only the quadratic terms yields

$$
\omega_{L, T}^{2}(q)=\left(a_{2} \pm b_{2}\right)|q|^{2}
$$

where the $+(-)$ sign goes with $\omega_{L}^{2}\left(\omega_{T}^{2}\right)$. The normal modes are

$$
\begin{aligned}
& u_{L}(q)=\frac{1}{\sqrt{2}|q|}\left(q^{*} u_{1}(q)+q u_{2}(q)\right) \\
& u_{T}(q)=-\frac{i}{\sqrt{2}|q|}\left(q^{*} u_{1}(q)-q u_{2}(q)\right)
\end{aligned}
$$

These represent longitudinal (LA) and transverse acoustic (TA) modes respectively. To see this, note that for $q a<<1$, we have

$$
u_{L}(q) \simeq \frac{1}{\sqrt{2}}\left(\begin{array}{c}
\cos \theta \\
\sin \theta \\
\cos \theta \\
\sin \theta
\end{array}\right), u_{T}(q) \simeq \frac{1}{\sqrt{2}}\left(\begin{array}{c}
-\sin \theta \\
\cos \theta \\
-\sin \theta \\
\cos \theta
\end{array}\right)
$$

where $q=|q| e^{i \theta}$, i.e. $\theta=\tan ^{-1}\left(q_{y} / q_{x}\right)$. In the long wavelength limit, the normal modes are completely independent of the coefficients in the dynamical matrix.

We can easily go beyond the quadratic approximation in $q$ valid in the long wavelength limit by keeping more terms in the expansions (21). Keeping just the first anisotropic contributions gives

$\omega_{L, T}^{2}(q)=\left(a_{2} \pm b_{2}\right)|q|^{2}+|q|^{4}\left(a_{4} \pm b_{4} \pm \tilde{b}_{4} \cos 6 \theta\right)+O\left(q^{6}\right)$
One can use perturbation theory to compute the leading corrections $u_{L, T} \rightarrow u_{L, T}+\delta u_{L, T}$ to the normal modes. Taking as perturbation the fourth order corrections to $D$

$$
\delta D=\left(\begin{array}{cc}
a_{4}|q|^{4} & b_{4}|q|^{2} q^{* 2}+\tilde{b}_{4} q^{4} \\
b_{4}|q|^{2} q^{2}+\tilde{b}_{4} q^{* 4} & a_{4}|q|^{4}
\end{array}\right)
$$

yields

$$
\begin{aligned}
& \delta u_{L} \simeq \frac{u_{T}^{\dagger} \delta D u_{L}}{\omega_{L}^{2}-\omega_{T}^{2}} u_{T} \simeq-\frac{\tilde{b}_{4}}{2 b_{2}}|q|^{2} \sin 6 \theta u_{T} \\
& \delta u_{T} \simeq \frac{u_{L}^{\dagger} \delta D u_{T}}{\omega_{T}^{2}-\omega_{L}^{2}} u_{L} \simeq \frac{\tilde{b}_{4}}{2 b_{2}}|q|^{2} \sin 6 \theta u_{L}
\end{aligned}
$$

Thus, beyond the long wavelength approximation the normal modes are not purely transverse or longitudinal, and (27) shows that this longitudinal-transverse (L-T) mixing is an $O\left(q^{2}\right)$ anisotropic effect.

The solution to the eigenvalue problem for the optical modes is very similar. The elements of the matrix $D^{\prime}(q)$ satisfy the same constraints (18), but the diagonal elements need not vanish for $q=0$. Keeping just the quadratic contributions

$$
D^{\prime}(q)=\left(\begin{array}{cc}
\omega_{0}^{2}+a_{2}^{\prime}|q|^{2} & b_{2}^{\prime} q^{* 2} \\
b_{2}^{\prime} q^{2} & \omega_{0}^{2}+a_{2}^{\prime}|q|^{2}
\end{array}\right)
$$

we find that the optical normal modes $u_{L}^{\prime}$ and $u_{T}^{\prime}$ are given by (23) in terms of the primed modes $u_{i}^{\prime}$, with normal frequencies

$$
\omega_{L, T}^{\prime 2}(q)=\omega_{0}^{2}+\left(a_{2}^{\prime} \pm b_{2}^{\prime}\right)|q|^{2}
$$

Similarly, L-T mixing for optical modes is still given by (27) if one uses primed constants.

We can also study acoustic-optical (A-O) mixing, which is induced by the matrix $M$. For example, keeping only the leading contributions to $M$ (see the Appendix B for details.)

$$
M(q) \simeq e_{1}\left(\begin{array}{cc}
0 & q \\
-q^{*} & 0
\end{array}\right)
$$

and using perturbation theory yields the following corrections to the LA and TA modes

$$
\begin{aligned}
& \delta u_{L} \simeq \frac{i e_{1}}{\omega_{0}^{2}}|q|\left(\sin 3 \theta u_{L}^{\prime}+\cos 3 \theta u_{T}^{\prime}\right) \\
& \delta u_{T} \simeq \frac{i e_{1}}{\omega_{0}^{2}}|q|\left(\cos 3 \theta u_{L}^{\prime}-\sin 3 \theta u_{T}^{\prime}\right)
\end{aligned}
$$

Thus A-O mixing is an $O\left(q / \omega_{0}^{2}\right)$ anisotropic effect. Exchanging primed and unprimed modes in (31) gives the corrections to the optical modes. This mixing induces a common shift in the frequencies of the acoustic modes

$$
\delta \omega_{L}^{2}=\delta \omega_{T}^{2} \simeq-\frac{e_{1}^{2}}{\omega_{0}^{2}}|q|^{2}
$$

while the opposite shift is induced in the optical frequencies. 


\section{ELECTRON-PHONON COUPLINGS}

In this section we use a similar strategy to obtain the most general interaction hamiltonian compatible with the symmetries of the system. We consider an electronphonon interaction hamiltonian of the form

$$
\begin{gathered}
H_{e-p h}=\sum_{i j, k, q} H_{i j}(k, q, \mathbf{Q}) c_{i+}^{\dagger}(k+q / 2) c_{j+}(k-q / 2) \\
+\sum_{i j, k, q} H_{-i j}(k, q, \mathbf{Q}) c_{i-}^{\dagger}(k+q / 2) c_{j-}(k-q / 2)
\end{gathered}
$$

where $c_{i \pm}(k)$ annihilates an electron in the state $\Phi_{i \pm}(k)$ given by (8) and $\mathbf{Q}=\left\{Q, Q^{\prime}\right\}$ stands for the four symmetry-adapted coordinates in (3). The somewhat unusual parametrization of initial and final electron momenta $(k \mp q / 2)$ simplifies the form of the constraints. The matrix element is given by

$$
H_{i j}(k, q, \mathbf{Q})=\left\langle\Phi_{i+}\left(k+\frac{q}{2}\right)\left|V_{e i}\right| \Phi_{j+}\left(k-\frac{q}{2}\right)\right\rangle
$$

where $V_{e i}$ is the electron-ion potential. Since the two Fermi points are related both by time-reversal and by $C_{2}$, either symmetry can be used to obtain the form of the hamiltonian near $\vec{K}_{2}=-\vec{K}_{1}$, giving

$$
H_{-}(k, q, \mathbf{Q})=H^{*}(-k,-q, \mathbf{Q})
$$

where

$$
H_{-i j}(k, q, \mathbf{Q}) \equiv\left\langle\Phi_{i-}(k+q / 2)\left|V_{e i}\right| \Phi_{j-}(k-q / 2)\right\rangle
$$

Thus, we can restrict ourselves to the hamiltonian in the vicinity of $\vec{K}_{1}$.

On the other hand, the combined symmetry $C_{2} \mathcal{T}$ (10) leaves the two Fermi points invariant and can be used to impose a constraint on $H\left(k, q, C_{2} \mathbf{Q}\right)$

$$
H\left(k, q, C_{2} \mathbf{Q}\right)=\sigma_{x} H^{*}(k, q, \mathbf{Q}) \sigma_{x}
$$

which is solved by

$$
H(k, q, \mathbf{Q})=\left(\begin{array}{cc}
\alpha(k, q, \mathbf{Q}) & \beta(k, q, \mathbf{Q}) \\
\beta^{*}\left(k, q, C_{2} \mathbf{Q}\right) & \alpha^{*}\left(k, q, C_{2} \mathbf{Q}\right)
\end{array}\right)
$$

where $\alpha$ and $\beta$ are arbitrary complex functions. Note that hermiticity of the hamiltonian does not imply reality for $\alpha$. The point is that the matrix $H\left(k, q, C_{2} \mathbf{Q}\right)$ connects initial and final states with different momenta, and instead we have

$$
\begin{array}{r}
H_{i j}(k, q, \mathbf{Q})=\left\langle\Phi_{i+}\left(k+\frac{q}{2}\right)\left|V_{e i}\right| \Phi_{j+}\left(k-\frac{q}{2}\right)\right\rangle \\
=\left\langle\Phi_{j+}\left(k-\frac{q}{2}\right)\left|V_{e i}\right| \Phi_{i+}\left(k+\frac{q}{2}\right)\right\rangle^{*}=H_{j i}^{*}(k,-q, \mathbf{Q})
\end{array}
$$

which implies

$$
\begin{aligned}
\alpha(k,-q, \mathbf{Q}) & =\alpha^{*}(k, q, \mathbf{Q}) \\
\beta(k,-q, \mathbf{Q}) & =\beta\left(k, q, C_{2} \mathbf{Q}\right)
\end{aligned}
$$

Finally, we must impose invariance under the little point group $C_{3 v}$

$$
T^{\dagger}(g) H(k, q, \mathbf{Q}) T(g)=H(g k, g q, g \mathbf{Q})
$$

where $T(g)$ is given by (5). The hamiltonian (38) together with the constraints (40,41) contains the most general interactions beween electrons and in-plane phonons compatible space group and time reversal symmetries. These constraints are analyzed in detail in Appendix C, where the general solution for the interaction hamiltonian is found in the form

$$
H(k, q, \mathbf{Q})=H(k, q, Q)+H^{\prime}\left(k, q, Q^{\prime}\right)
$$

with

$$
\begin{gathered}
H(k, q, Q)=i\left(\begin{array}{cc}
f(k, q) Q_{1}(q)-f(k,-q)^{*} Q_{2}(q) & g_{1}(k, q) Q_{1}(q)+g_{2}(k, q) Q_{2}(q) \\
g_{2}(k, q)^{*} Q_{1}(q)+g_{1}(k, q)^{*} Q_{2}(q) & -f(k,-q) Q_{1}(q)+f(k, q)^{*} Q_{2}(q)
\end{array}\right) \\
H^{\prime}\left(k, q, Q^{\prime}\right)=i\left(\begin{array}{cc}
f^{\prime}(k, q) Q_{1}^{\prime}(q)-f^{\prime}(k,-q)^{*} Q_{2}^{\prime}(q) & g_{1}^{\prime}(k, q) Q_{1}^{\prime}(q)+g_{2}^{\prime}(k, q) Q_{2}^{\prime}(q) \\
-g_{2}^{\prime}(k, q)^{*} Q_{1}^{\prime}(q)-g_{1}^{\prime}(k, q)^{*} Q_{2}^{\prime}(q) & f^{\prime}(k,-q) Q_{1}^{\prime}(q)-f^{\prime}(k, q)^{*} Q_{2}^{\prime}(q)
\end{array}\right)
\end{gathered}
$$

The functions entering the hamiltonian must satisfy some simple conditions. In particular the functions $f$, $f^{\prime}, g_{2}$, and $g_{2}^{\prime}$ must satisfy identical constraints, which we write only for $f$

$$
f(\omega k, \omega q)=\omega f(k, q), f\left(k^{*}, q^{*}\right)=f(k, q)^{*}
$$


whereas the conditions on $g_{1}$ and $g_{1}^{\prime}$ are

$$
g_{1}(\omega k, \omega q)=g_{1}(k, q), g_{1}\left(k^{*}, q^{*}\right)=g_{1}(k, q)^{*}
$$

Besides, we must have

$$
g_{i}(k,-q)=-g_{i}(k, q), \quad g_{i}^{\prime}(k,-q)=g_{i}^{\prime}(k, q)
$$

Since $\omega^{3}=1$, one immediately sees that any combination of monomials of the form

$$
k^{n} k^{* n^{\prime}} q^{m} q^{* m^{\prime}}
$$

with real coefficients and $n-n^{\prime}+m-m^{\prime}=1 \bmod 3$ will satisfy (44). Imposing instead $n-n^{\prime}+m-m^{\prime}=0 \bmod 3$ we will get solutions to (45). Finally, the conditions (46) are satisfied by taking $m+m^{\prime}$ odd (even) for $g_{i}\left(g_{i}^{\prime}\right)$.

Eq. (43) together with the comments around eq. (47) can be used to expand the interaction hamiltonian to any order in $q$ and $k$, giving all possible in-plane phononelectron couplings compatible with the symmetries of monolayer graphene, and are the main result in this paper. For the rest of this section we will study the long wavelength limit and some leading corrections.

Consider first the coupling to acoustic phonons. Taking into account that the interaction should vanish for $q \rightarrow 0$, we can immediately write the leading contributions to the functions $f, g_{1}, g_{2}$

$$
f(k, q) \approx \sqrt{2} \alpha_{1} q, g_{1}(k, q) \approx 0, g_{2}(k, q) \approx \sqrt{2} \beta_{1} q
$$

where $\alpha_{1}$ and $\beta_{1}$ are real constants and the $\sqrt{2}$ has been introduced for later convenience. This gives

$$
\begin{aligned}
& H(k, q, Q) \approx \\
& i \sqrt{2}\left(\begin{array}{cc}
\alpha_{1}\left(q Q_{1}(q)+q^{*} Q_{2}(q)\right) & \beta_{1} q Q_{2}(q) \\
\beta_{1} q^{*} Q_{1}(q) & \alpha_{1}\left(q Q_{1}(q)+q^{*} Q_{2}(q)\right)
\end{array}\right)
\end{aligned}
$$

For some physical applications it may be more convenient to express the hamiltonian as a function of normal rather than symmetry-adapted coordinates. As we know from the previous section, the longitudinal and transverse modes in (23) are a good approximation to the actual normal modes in the long-wavelength limit. Using

$$
Q_{1}=\frac{q^{*}}{\sqrt{2}|q|}\left(Q_{L}-i Q_{T}\right) \quad, \quad Q_{2}=\frac{q}{\sqrt{2}|q|}\left(Q_{L}+i Q_{T}\right)
$$

the interaction hamiltonian takes the following form

$$
\begin{aligned}
& H(k, q, Q) \approx \\
& i|q|\left(\begin{array}{cc}
2 \alpha_{1} Q_{L} & \beta_{1} e^{2 i \theta}\left(Q_{L}+i Q_{T}\right) \\
\beta_{1} e^{-2 i \theta}\left(Q_{L}-i Q_{T}\right) & 2 \alpha_{1} Q_{L}
\end{array}\right)
\end{aligned}
$$

where $\theta=\tan ^{-1}\left(q_{y} / q_{x}\right)$ is the phase of $q=|q| e^{i \theta}$. This shows that TA phonons do not couple diagonally to electrons in the long-wavelength limit, which is parametrized by the two real coulplings $\alpha_{1}$ and $\beta_{1}$. This form of the hamiltonian can be used to write (33) in terms of phonon creation and annihilation operators, $\frac{6}{-}$ with $Q_{L, T}(q)=\left(2 \omega_{L, T}\right)^{-1 / 2}\left(a_{L, T}(q)+a_{L, T}^{\dagger}(-q)\right)$.

Note that even if one is primarily interested in acoustic phonons, because of the acoustic-optical mixing discussed in the last section, one may have to consider the couplings of electrons to optical modes as well. Since the couplings to optical modes do not have to vanish as $q \rightarrow 0$, the leading contributions to the hamiltonian are independent of $q$. These are found to be

$$
\begin{aligned}
& H\left(k, q, Q^{\prime}\right) \approx i \sqrt{2} \beta_{1}^{\prime}\left(\begin{array}{cc}
0 & Q_{1}^{\prime} \\
-Q_{2}^{\prime} & 0
\end{array}\right) \\
= & i \beta_{1}^{\prime}\left(\begin{array}{cc}
0 & e^{-i \theta}\left(Q_{L}^{\prime}-i Q_{T}^{\prime}\right) \\
-e^{i \theta}\left(Q_{L}^{\prime}+i Q_{T}^{\prime}\right) & 0
\end{array}\right)
\end{aligned}
$$

where $\beta_{1}^{\prime}$ is a real constant.

We can easily include higher powers of the momenta. For instance, for acoustic phonons the contribution of order $O\left(q^{2}\right)$ is parametrized by a single real constant $\alpha_{2}$ and is purely diagonal and proportional to the Pauli matrix $\sigma_{z}$

$$
\Delta H(k, q, Q)=\alpha_{2}|q|^{2}\left(Q_{L} \sin 3 \theta+Q_{T} \cos 3 \theta\right) \sigma_{z}
$$

This is qualitatively different from the leading contribution (51) due to the anisotropy in the strength of the electron-phonon couplings and the diagonal contribution of TA phonons. The contribution of order $O(q)$ for optical phonons is proportional to the unit matrix

$$
\Delta H^{\prime}\left(k, q, Q^{\prime}\right)=i \alpha_{2}^{\prime}|q| Q_{L}^{\prime} \mathbf{1}
$$

and there is also an $O(k)$ term given by

$$
\Delta H^{\prime}\left(k, q, Q^{\prime}\right)=\alpha_{3}^{\prime}|k|\left(Q_{L}^{\prime} \sin (\varphi-\theta)+Q_{T}^{\prime} \cos (\varphi-\theta)\right) \mathbf{1}
$$

where $\varphi=\tan ^{-1}\left(k_{y} / k_{x}\right)$. The contributions of order $O(q k)$ are more complicated and depend on five real constants. Note that as we move away from the $\Gamma$-point the LA and TA modes given by (23) are no longer a good approximation to the normal modes of the system, and one has to take into account the $\mathrm{L}-\mathrm{T}$ and $\mathrm{A}-\mathrm{O}$ mixings considered in the previous section.

Electron-phonon interactions can be rewritten in terms of electrons and holes. The eigenstates for the electron hamiltonian (2) are given by

$$
\Phi_{ \pm}(k)=\frac{1}{\sqrt{2}}\left(e^{-i \varphi / 2} \Phi_{1}(k) \pm e^{i \varphi / 2} \Phi_{2}(k)\right)
$$

and this can be used to obtain the matrix elements between electron eigenstates, with $H_{++}$and $H_{--}\left(H_{+-}\right.$ and $H_{-+}$) corresponding to intraband (interband ) transitions. Of course, if we decide to include higher powers of $k$ in the electron-phonon hamiltonian, for consistency we must also go beyond the low-energy Dirac hamiltonian (2). The corrections to the electronic hamiltonian are fixed by symmetry. Note, in particular, that 
the electronic hamiltonian can be considered as the Qindependent part of (34). Then (38) reduces to

$$
H(k)=\left(\begin{array}{cc}
\alpha_{0}(k) & \beta_{0}(k) \\
\beta_{0}^{*}(k) & \alpha_{0}^{*}(k)
\end{array}\right)
$$

where hermiticity (40) implies that $\alpha_{0}$ is real. The little group constraints (C1) simplify to

$$
\begin{gathered}
C_{3}^{+}: \alpha_{0}(\omega k)=\alpha_{0}(k), \quad \beta_{0}(\omega k)=\omega^{*} \beta_{0}(k) \\
\sigma_{v 1}: \alpha_{0}\left(k^{*}\right)=\alpha_{0}(k)^{*}, \quad \beta_{0}\left(k^{*}\right)=\beta_{0}(k)^{*}
\end{gathered}
$$

which can be easily solved to any order in $k$. Taking the chemical potential at half filling as the origin of energies and $v_{F}=1$, the first few terms are

$$
\begin{aligned}
\alpha_{0}(k) & =\alpha_{0,2}|k|^{2}+\alpha_{0,3}\left(k^{3}+k^{* 3}\right)+\ldots \\
\beta_{0}(k) & =k^{*}+\beta_{0,2} k^{2}+\beta_{0,3}|k|^{2} k^{*}+\ldots
\end{aligned}
$$

where all the constants are real. The eigenstates are still given by (56) if one replaces the phase of $k$ by the phase of $\beta_{0}^{*}(k)$.

\section{ELASTIC STRAINS AS EFFECTIVE ELECTROMAGNETIC FIELDS}

Here we show that the couplings of long wavelength phonons to electrons have some similarities - and differences - with those of the scalar and vector potentials for the electromagnetic field. We show, in particular, that both in-plane and out-of-plane strains can mimic some of the effects of electric and magnetic fields.

The minimal coupling prescription $k_{i} \rightarrow k_{i}+A_{i}$ on (2) gives

$$
\begin{aligned}
H(k) & =\left(\begin{array}{cc}
\Phi & k^{*}+A^{*} \\
k+A & \Phi
\end{array}\right) \\
H_{-}(k) & =\left(\begin{array}{cc}
\Phi & -k-A \\
-k^{*}-A^{*} & \Phi
\end{array}\right)
\end{aligned}
$$

where $A=A_{x}+i A_{y}$ is the vector potential in complex notation and $\Phi$ is the scalar (electric) potential. For the purposes of this section it is convenient to change from the circularly polarized modes of table I to linear modes $u_{x}, u_{y}$

$$
\begin{array}{rlrl}
u_{1} & =\frac{1}{\sqrt{2}}\left(u_{x}+i u_{y}\right) \quad, \quad u_{2}=\frac{1}{\sqrt{2}}\left(u_{x}-i u_{y}\right) \Rightarrow \\
Q_{1}=\frac{1}{\sqrt{2}}\left(Q_{x}-i Q_{y}\right) & , \quad Q_{2} & =\frac{1}{\sqrt{2}}\left(Q_{x}+i Q_{y}\right)
\end{array}
$$

Then, the acoustic hamiltonian (49) can be rewritten by using

$$
\begin{array}{r}
i \sqrt{2}\left(q Q_{1}+q^{*} Q_{2}\right)=2 i\left(q_{x} Q_{x}+q_{y} Q_{y}\right) \\
i \sqrt{2} q Q_{2}=2 i\left(q_{x} Q_{x}-q_{y} Q_{y}\right)-2\left(q_{x} Q_{y}+q_{y} Q_{x}\right) \\
i \sqrt{2} q^{*} Q_{1}=2 i\left(q_{x} Q_{x}-q_{y} Q_{y}\right)+2\left(q_{x} Q_{y}+q_{y} Q_{x}\right)
\end{array}
$$

This agrees with the hamiltonian given in ref ${ }^{15}$ where the off-diagonal elements (second and third lines) were obtained by expanding a phonon-modulated hopping, while the magnitude of the diagonal element, known as 'deformation potential', was estimated in a nearly free electron model. Our derivation shows that both diagonal and offdiagonal terms are uniquely determined by symmetry.

Taking Fourier transforms with $i q_{i} \rightarrow \partial_{i}$ and comparing with (60) finally yields

$$
\begin{array}{r}
\Phi\left(K_{1}\right)=\Phi\left(K_{2}\right)=2 \alpha_{1}\left(\partial_{x} Q_{x}+\partial_{y} Q_{y}\right) \\
A_{x}\left(K_{1}\right)=-A_{x}\left(K_{2}\right)=2 \beta_{1}\left(\partial_{x} Q_{x}-\partial_{y} Q_{y}\right) \\
A_{y}\left(K_{1}\right)=-A_{y}\left(K_{2}\right)=-2 \beta_{1}\left(\partial_{x} Q_{y}+\partial_{y} Q_{x}\right)
\end{array}
$$

where we have used (35) to obtain the electron-phonon hamiltonian around $\vec{K}_{2}$. These effective fields can be written in terms of elastic strains with the usual definitions

$$
u_{x x}=\partial_{x} Q_{x}, u_{y y}=\partial_{y} Q_{y}, 2 u_{x y}=\partial_{x} Q_{y}+\partial_{y} Q_{x}
$$

Thus we have the remarkable result that, around each Fermi point, static strains can mimic the effects of external electric and magnetic fields.

We also see that the couplings of electrons near the two Fermi points are identical for the scalar potential, but differ by a sign for the vector potential. Thus, for static strains they will experience the same 'electrostatic' field, but opposite 'magnetic' fields given by

$$
\begin{aligned}
B_{z}\left(K_{1}\right) & =-B_{z}\left(K_{2}\right)=\partial_{x} A_{y}\left(K_{1}\right)-\partial_{y} A_{x}\left(K_{1}\right) \\
& =-2 \beta_{1}\left[\left(\partial_{x}^{2}-\partial_{y}^{2}\right) Q_{y}+2 \partial_{x} \partial_{y} Q_{x}\right] \\
& =-2 \beta_{1}\left[2 \partial_{x} u_{x y}+\partial_{y}\left(u_{x x}-u_{y y}\right)\right]
\end{aligned}
$$

We now address the following question: Is the (partial) identification between elastic strains and effective electromagnetic fields peculiar to graphene alone, or can we expect to find it in other systems? The first observation is straightforward: The minimal coupling prescription $\vec{k} \rightarrow \vec{k}+\vec{A}$ gives rise to the required type of interactions for the vector potential only if the electron system satisfies the Dirac equation. An ordinary non-relativistic equation would produce terms of the type $\vec{k} \cdot \vec{A}$, where $\vec{k}$ is the electron momentum, unlike the leading phonon couplings (49), which are $k$-independent.

But even with Dirac points, further conditions have to be satisfied. The effective fields (63) associated to acoustic phonons are of the form $\partial_{i} Q_{j}$, where both $\partial_{i}$ and $Q_{i}$ belong to the vector representation $V$. The vector potential also belongs to $V$. In the continuum, $V \times V$ and $V$ have opposite parities, and $\partial_{i} Q_{j}$ and $A$ can not transform equivalently. Thus, the possibility of describing elastic strains as effective fields is a lattice effect. More concretely, we need a lattice without inversion symmetry.

But even on a lattice this identification can be partial at best. The reason is that the vector potential is odd under time reversal, whereas phonons and their derivatives are even. Since time reversal takes $\vec{K}$ to $-\vec{K}$, as long as 
$-\vec{K}$ is not equivalent to $\vec{K}$, we can still hope for a partial identification valid around individual Fermi points. This will be possible only if the representations $V$ and $V \times V$ of the little group $G_{\vec{K}}$ have at least one irreducible representation in common, i.e., if $V^{3}$ contains the identity (or trivial) representation.

We now discuss how these conditions are met by monolaye graphene. The littele group is $\hat{G}_{\vec{K}_{1}}=C_{3 v}$ with a vector representation that decomposes according to $\underline{2}$

$$
V=A_{1}(z)+E(x, y)
$$

For in-plane modes only $E$ is relevant, and the use of elementary group-theory techniques ${ }^{1,2}$ yields

$$
\begin{aligned}
E \times E & =A_{1}\left(\partial_{x} Q_{x}+\partial_{y} Q_{y}\right)+A_{2}\left(\partial_{x} Q_{y}-\partial_{y} Q_{x}\right) \\
& +E\left(\partial_{y} Q_{y}-\partial_{x} Q_{x}, \partial_{x} Q_{y}+\partial_{y} Q_{x}\right)
\end{aligned}
$$

Here we recognize the L.H.S. of (63) as the basis for the irreducible representations $A_{1}$ and $E$. But the fact that the vector potential $\left(A_{x}, A_{y}\right)$ and certain components of the strain tensor transform equivalently under the little point group $C_{3 v}$ is not sufficient to guarantee that elastic strains can mimic a magnetic field around each Fermi point, for they could still couple to the two atoms in the unit cell with different signs. By (10), the two atoms are exchaged under $C_{2} \mathcal{T}$. Now, the vector potential is odd under $C_{2}$ and $\mathcal{T}$, whereas the the in-plane components of the elastic strain are even under both symmetries, and the two minus signs cancell each other. However, electrons at the two Fermi points see effective vector potentials which differ by the sign. On the other hand, the scalar potential $\Phi$ belongs to the trivial representation $A_{1}$ and is even under time reversal. As a consequence, the effective scalar potential takes the same sign on $\vec{K}_{1}$ and $\vec{K}_{2}=-\vec{K}_{1}$.

This analysis can be extended to include the effects of so-called ripples or long wavelength deformations perpendicular to the grapehene sheet. Such ripples make the two dimensional graphene sheet thermodinamically stable and have been recently observed in individually suspended sheets ${ }^{29}$. Although we have restricted ourselves to in-plane modes, acoustic out-of-plane modes can be easily incorporated in the long wavelength limit (see Section 6 for the possibility of a more general analysis):

According to (66) a vertical displacement $Q_{z}$ belongs to the IR $A_{1}$ of $C_{3 v}$. On the other hand, $Q_{z}$ is odd under reflection by the horizontal plane $\sigma_{h}$, whereas electronic wavefunction bilinears are necessarily even. This forces us to consider quadratic functions of $Q_{z}$. By translation invariance along the $z$ axis only derivatives of $Q_{z}$ are acceptable, and we have to consider quadratic functions of $\partial_{x} Q_{z}$ and $\partial_{y} Q_{z}$. The basis for the IRs $A_{1}$ and $E$ are now given by

$$
\begin{array}{r}
A_{1}\left(\left(\partial_{x} Q_{z}\right)^{2}+\left(\partial_{y} Q_{z}\right)^{2}\right) \\
E\left(\left(\partial_{y} Q_{z}\right)^{2}-\left(\partial_{x} Q_{z}\right)^{2}, 2 \partial_{x} Q_{z} \partial_{y} Q_{z}\right)
\end{array}
$$

The discussion after Eq. (67) applies also in this case, implying that the couplings of electrons near the two Fermi points are identical for the scalar potential, but differ by a sign for the vector potential. Thus, the associated effective fields are given by

$$
\begin{array}{r}
\Phi\left(K_{1}\right)=\Phi\left(K_{2}\right)=\gamma\left(\left(\partial_{x} Q_{z}\right)^{2}+\left(\partial_{y} Q_{z}\right)^{2}\right) \\
A_{x}\left(K_{1}\right)=-A_{x}\left(K_{2}\right)=\delta\left(\left(\partial_{y} Q_{z}\right)^{2}-\left(\partial_{x} Q_{z}\right)^{2}\right) \\
A_{y}\left(K_{1}\right)=-A_{y}\left(K_{2}\right)=2 \delta \partial_{x} Q_{z} \partial_{y} Q_{z}
\end{array}
$$

where $\gamma$ and $\delta$ are real coupling constants.

We close this section by noting that frozen optical modes can also give rise to effective magnetic fields. 33 Indeed, the substitution of (61) in (52) gives

$$
\begin{aligned}
H\left(k, q, Q^{\prime}\right) & =i \sqrt{2} \beta_{1}^{\prime}\left(\begin{array}{cc}
0 & Q_{1}^{\prime} \\
-Q_{2}^{\prime} & 0
\end{array}\right) \\
& =\beta_{1}^{\prime}\left(\begin{array}{cc}
0 & Q_{y}^{\prime}+i Q_{x}^{\prime} \\
Q_{y}^{\prime}-i Q_{x}^{\prime} & 0
\end{array}\right)
\end{aligned}
$$

Comparing with the minimally coupled hamiltonians (60) yields the identifications

$$
\begin{aligned}
& A_{x}^{\prime}\left(K_{1}\right)=-A_{x}^{\prime}\left(K_{2}\right)=\beta_{1}^{\prime} Q_{y}^{\prime} \\
& A_{y}^{\prime}\left(K_{1}\right)=-A_{y}^{\prime}\left(K_{2}\right)=-\beta_{1}^{\prime} Q_{x}^{\prime}
\end{aligned}
$$

and the magnetic fields

$$
B_{z}^{\prime}\left(K_{1}\right)=-B_{z}^{\prime}\left(K_{2}\right)=-\beta_{1}^{\prime}\left(\partial_{x} Q_{x}^{\prime}+\partial_{y} Q_{y}^{\prime}\right)
$$

\section{DISCUSSION}

In this paper we have exploited the fact that, for many physical processes of interest in monolayer graphene, the wavevectors of electrons and phonons lie near points of high symmetry in the Brillouin zone. Even though the little groups for non-vanishing $k$ and $q$ are generically trivial, the proximity to points of high symmetry impose strong constraints on the series expansions of observables around them. This is analogous to the situation in Landau's theory ${ }^{\underline{1}}$ of second order phase transitions, where the dependence on the order parameter of observables in the low symmetry phase is determined by the space group of the high symmetry phase. Here, $q$ and $k$ play the role of order parameters, 'breaking' the symmetries of the $\Gamma$ and Fermi points respectively. As a consequence, the method presented in this paper may be useful in other systems with Fermi points, such as multilayer graphene. ${ }^{22,26,27,28}$ Semimetals with small electron and hole pockets around high symmetry points are also good candidates.

Note that, in spite of the fact that we use tight-binding Bloch functions as our starting point, our results are model-independent. The reason is that only the symmetry properties of the wavefunctions are used, and the general electronic hamiltonian (57) describes any doublet of states transforming according to the small representation $E$ of the little group.

The relatively involved constraints 40410 on the electron-phonon hamiltonian are reduced to extremely 
simple conditions on the few functions which appear in the general solution (43) in Section 4 - so simple, indeed, that they can be solved explicitly. In the process the general functions $\alpha$ and $\beta$ are written as linear combinations of the symmetry-adapted coordinates. By dropping the linearity assumption we could extend our approach to multiphonon processes. One can easily check that the constraints (4041) are still valid for nonlinear functions depending on several modes and momenta, with

$$
\alpha(k, q, \mathbf{Q}) \rightarrow \alpha\left(k,\left\{q_{l}\right\},\left\{\mathbf{Q}_{l}\left(q_{l}\right)\right\}\right)
$$

and other obvious replacements. Note that at the nonlinear level out-of-plane modes have to be included for consistency, and the whole symmmetry $D_{6 h}$ rather than $C_{6 v}$ has to be used. The analysis becomes more involved and will be the object of future work.

Inspired by a mechanism first proposed in ref ${ }^{30}$ for the case of dislocations in multivalley conductors, effective magnetic fields induced by elastic distorsions have been recently suggested as a way to explain the strong suppression of weak localization in graphene $\stackrel{31,32}{\text { This }}$ differs from our results in Section 5 in that they consider effective gauge fields which are quadratic in the out-ofplane modes. Our analysis in the previous section shows that in-plane modes give rise to effective fields which are linear in the strains. For physical applications of the treatment in Section 5 to dislocations and other deffects see ref 33

Our method can be easily extended to accomodate external fields. For instance, in the presence of an electric field along the $z$ axis, out-of-plane phonons will couple linearly to the electrons. This may be an externally applied electric field, or the effect of a substrate which breaks the symmetry under reflections on the sample plane. The recently observed ripples in suspended graphene sheets ${ }^{29}$ also break the reflection symmetry $\sigma_{h}$ and can play the role of a 'background field', inducing new electron-phonon interactions.

\section{Acknowledgments}

It is a pleasure to thank F. Guinea for motivating this work and for very useful discussions and suggestions, particularly in reference to Section 5. This work has been supported in part by the Spanish Science Ministry under Grant FPA2005-04823.

\section{APPENDIX A: TRANSFORMATIONS OF MODES AND WAVEFUNCTIONS}

The action of the generators of $C_{6 v}$ on the electron and phonon wavevectors is given by the vector representation. This is simpler in complex notation

$$
C_{3}^{+} q=\omega q \quad, \quad \sigma_{v 1} q=q^{*} \quad, \quad C_{2} q=-q \quad, \quad \omega \equiv e^{2 \pi i / 3}
$$

where $q=q_{x}+i q_{y}$.

Careful inspection of Table 1 shows that the generator $\mathrm{C}_{3}^{+}$acts identically on primed and unprimed symmetryadapted modes, namely

$$
C_{3}^{+}: u_{1}(q) \rightarrow \omega^{*} u_{1}(\omega q) \quad, \quad u_{2}(q) \rightarrow \omega u_{2}(\omega q)
$$

where $\omega \equiv \exp (2 \pi i / 3)$. The actions of $\sigma_{v 1}$ and $C_{2}$, however, are different for the two types of modes

$$
\begin{aligned}
\sigma_{v 1}: & u_{1}(q) \rightarrow u_{2}\left(q^{*}\right), \quad u_{2}(q) \rightarrow u_{1}\left(q^{*}\right) \\
& u_{1}^{\prime}(q) \rightarrow-u_{2}^{\prime}\left(q^{*}\right), \quad u_{2}^{\prime}(q) \rightarrow-u_{1}^{\prime}\left(q^{*}\right) \\
C_{2}: & u_{i}(q) \rightarrow-u_{i}(-q) \quad, \quad u_{i}^{\prime}(q) \rightarrow u_{i}^{\prime}(-q)
\end{aligned}
$$

Correspondingly, the symmetry-adapted coordinates $Q_{i}$ and $Q_{i}^{\prime}$ in (3) have transformation properties

$$
g Q(g q)=T(g) Q(q) \quad, \quad g Q^{\prime}(g q)=T^{\prime}(g) Q^{\prime}(q)
$$

where $T^{\prime}\left(C_{2}\right)=-T\left(C_{2}\right)=\mathbf{1}$ is the unit matrix, and

$$
\begin{gathered}
T\left(\sigma_{v 1}\right)=-T^{\prime}\left(\sigma_{v 1}\right)=\sigma_{x}=\left(\begin{array}{ll}
0 & 1 \\
1 & 0
\end{array}\right) \\
T\left(C_{3}^{+}\right)=T^{\prime}\left(C_{3}^{+}\right)=\Omega \equiv\left(\begin{array}{cc}
\omega^{*} & 0 \\
0 & \omega
\end{array}\right)
\end{gathered}
$$

Note that at the $\Gamma$-point the unprimed (primed) modes transform according to the two dimensional representation $E_{1}\left(E_{2}\right)$ of $C_{6 v}$.

The action of the generators of the little group $C_{3 v}$ on the electronic wavefunctions is given by

$$
\begin{aligned}
C_{3}^{+}: & \Phi_{1+}(k) \rightarrow \omega \Phi_{1+}(\omega k), \Phi_{2+}(k) \rightarrow \omega^{*} \Phi_{2+}(\omega k) \\
& \Phi_{1-}(k) \rightarrow \omega^{*} \Phi_{1-}(\omega k), \Phi_{2-}(k) \rightarrow \omega \Phi_{2-}(\omega k) \\
\sigma_{v 1}: & \Phi_{1+}(k) \leftrightarrow \Phi_{2+}\left(k^{*}\right), \Phi_{1-}(k) \leftrightarrow \Phi_{2-}\left(k^{*}\right) \quad(\mathrm{A} 6)
\end{aligned}
$$

The binary axis $C_{2}$ connects the two Fermi points

$$
C_{2}: \Phi_{1+}(k) \leftrightarrow \Phi_{2-}(-k), \Phi_{2+}(k) \leftrightarrow \Phi_{1-}(-k)
$$

Equations A6[A7) imply that, at the Fermi points $(k=0)$, the wavefunctions $\left\{\Phi_{i \pm}(0)\right\}$ form the basis for the 4-dimensional IR $E$ of the space group ${ }^{21}$ with star $\left\{\vec{K}_{1},-\vec{K}_{1}\right\}$.

\section{APPENDIX B: SYMMETRIES OF THE DYNAMICAL MATRIX}

Since according to (13) invariance under the generators of $C_{3 v}$ impose identical constraints on $D$ and $D^{\prime}$, we write these only for $D$

$$
C_{3}^{+}: D(\omega q)=\Omega D(q) \Omega^{*}, \sigma_{v 1}: D\left(q^{*}\right)=\sigma_{x} D(q) \sigma_{x}
$$

The nondiagonal block $M$ satisfies the same constraint under $C_{3}^{+}$, but gets an additional minus sign under $\sigma_{v 1}$

$$
\sigma_{v 1}: M\left(q^{*}\right)=-\sigma_{x} M(q) \sigma_{x}
$$


Invariance under $C_{2}$ implies that $D$ and $D^{\prime}$ are even functions of $q$, whereas $M$ is odd

$$
C_{2}: D(-q)=D(q), D^{\prime}(-q)=D^{\prime}(q), M(-q)=-M(q)
$$

Finally, time-reversal invariance (7) imposes the additional constraint

$$
D(-q)=\sigma_{x} D^{*}(q) \sigma_{x}
$$

which is also satisfied by $D^{\prime}$ and $M$. Combining this equation with (B1)-(B3) gives

$$
D\left(q^{*}\right)=D^{*}(q), D^{\prime}\left(q^{*}\right)=D^{* *}(q), M\left(q^{*}\right)=M^{*}(q)
$$

These constraints are solved by

$$
D(q)=\left(\begin{array}{cc}
a(q) & b(q) \\
b^{*}(q) & a(q)
\end{array}\right)
$$

where $a(q)$ and $b(q)$ are even functions of $q$ that satisfy

$$
\begin{aligned}
& a(\omega q)=a(q), b(\omega q)=\omega b(q) \\
& a\left(q^{*}\right)=a(q), b\left(q^{*}\right)=b^{*}(q)
\end{aligned}
$$

with identical solution for $D^{\prime}(q)$. For the off-diagonal block the solution is

$$
M(q)=\left(\begin{array}{cc}
d(q) & e(q) \\
-e\left(q^{*}\right) & -d\left(q^{*}\right)
\end{array}\right)
$$

where $d(q)$ and $e(q)$ are odd functions of $q$ that satisfy the following constraints

$$
\begin{array}{ccl}
d(\omega q)=d(q) & , & e(\omega q)=\omega e(q) \\
d\left(q^{*}\right)=d^{*}(q) & , \quad e\left(q^{*}\right)=e^{*}(q)
\end{array}
$$

These imply the expansions

$$
\begin{aligned}
& d(q)=d_{3} q^{3}+\tilde{d}_{3} q^{* 3}+O\left(q^{5}\right) \\
& e(q)=e_{1} q+e_{3}|q|^{2} q+O\left(q^{5}\right)
\end{aligned}
$$

where all the constants are real.

\section{APPENDIX C: LITTLE GROUP CONSTRAINTS ON THE ELECTRON-PHONON HAMILTONIAN}

Substitution of (38) into (41) yields the following constraints on the complex functions $\alpha$ and $\beta$

$$
\begin{aligned}
C_{3}^{+}: & \alpha\left(\omega k, \omega q, C_{3}^{+} \mathbf{Q}\right)=\alpha(k, q, \mathbf{Q}) \\
& \beta\left(\omega k, \omega q, C_{3}^{+} \mathbf{Q}\right)=\omega^{*} \beta(k, q, \mathbf{Q}) \\
\sigma_{v 1}: \quad & \alpha\left(k^{*}, q^{*}, \sigma_{v 1} \mathbf{Q}\right)=\alpha^{*}\left(k, q, C_{2} \mathbf{Q}\right) \\
& \beta\left(k^{*}, q^{*}, \sigma_{v 1} \mathbf{Q}\right)=\beta^{*}\left(k, q, C_{2} \mathbf{Q}\right)
\end{aligned}
$$

where the argument of $\mathbf{Q}$ is always the second one in the function, i.e., $\alpha\left(\omega k, \omega q, C_{3}^{+} \mathbf{Q}\right)$ actually stands for $\alpha\left(\omega k, \omega q, C_{3}^{+} \mathbf{Q}(\omega q)\right)$. The general solution to the constraints can be found by writing

$$
\begin{aligned}
& \alpha(k, q, \mathbf{Q})=\alpha(k, q, Q)+\alpha^{\prime}\left(k, q, Q^{\prime}\right) \\
& \beta(k, q, \mathbf{Q})=\beta(k, q, Q)+\beta^{\prime}\left(k, q, Q^{\prime}\right)
\end{aligned}
$$

with

$$
\begin{aligned}
& \alpha(k, q, Q)=i\left(f_{1}(k, q) Q_{1}(q)+f_{2}(k, q) Q_{2}(q)\right) \\
& \beta(k, q, Q)=i\left(g_{1}(k, q) Q_{1}(q)+g_{2}(k, q) Q_{2}(q)\right)
\end{aligned}
$$

and equivalent expressions for $\alpha^{\prime}$ and $\beta^{\prime}$ in terms of primed variables. Then, substitution of $\alpha$ into the hermiticity conditions (40) gives

$$
f_{2}(k, q)=-f_{1}(k,-q)^{*}
$$

which implies

$$
\alpha(k, q, Q)=i\left(f(k, q) Q_{1}(q)-f^{*}(k,-q) Q_{2}(q)\right)
$$

with identical results for $\alpha^{\prime}$. Imposing the conditions (40) on $\beta$ and $\beta^{\prime}$ gives

$$
g_{i}(k,-q)=-g_{i}(k, q) \quad, \quad g_{i}^{\prime}(k,-q)=g_{i}^{\prime}(k, q)
$$

Substitution into the little group constraints (C1) shows that the functions $f, f^{\prime}, g_{2}$, and $g_{2}^{\prime}$ must satisfy identical conditions, which we write only for $f$

$$
f(\omega k, \omega q)=\omega f(k, q), \quad f\left(k^{*}, q^{*}\right)=f(k, q)^{*}
$$

whereas the conditions on $g_{1}$ and $g_{1}^{\prime}$ are

$$
g_{1}(\omega k, \omega q)=g_{1}(k, q), g_{1}\left(k^{*}, q^{*}\right)=g_{1}(k, q)^{*}
$$

The general solution for the electron-phonon hamiltonian is then given by (42/43).
${ }^{1}$ G.Y. Lyubarskii, The Aplication of Group Theory in Physics (Pergamon, Oxford, 1960)
${ }^{2}$ C.J. Bradley and A.P. Cracknell, The Mathematical The- 
ory of Symmetry in Solids (Clarendon, Oxford, 1972)

3 A.A. Maradudin and S.H. Vosko, Rev. Mod. Phys. 40, 1 (1968)

4 Symmetry elements that do not belong to the little group can be used to relate observables at different points of the Brillouin zone, but they do not impose constraints on the form of observables at a particular point.

5 J.M. Ziman, Electrons and Phonons (Oxford, New YYork, 1960)

${ }^{6}$ G.D. Mahan, Many-Particle Physics, 2nd ed. (Plenum, New York, 1990)

7 S. Barisic, J. Labbe and J. Friedel, Phys. Rev. Lett. 25, 919 (1970)

8 L. Pietronero, S. Strassler, H.R. Zeller and M.J. Rice, Phys. Rev. B 22, 904 (1980)

9 R.A. Jishi, M.S. Dresselhaus and G. Dresselhaus, Phys. Rev. B 48, 11385 (1993)

10 G.D. Mahan and L.M. Woods, Phys. Rev. B 60, 5276 (1999)

11 K.S. Novoselov, A.K. Geim, S.V. Morozov, D. Jiang, Y. Zhang, S.V. Dubonos, I. V. Gregorievna and A.A. Firsov, Science 306, 666 (2004)

12 K.S. Novoselov, D. Jiang, T. Booth, V. Khotkevich, S.M. Morozov and A.K. Geim, Proc. Nat. Acad. Sci. U.S.A. 102, 10451(2005)

13 L.M. Woods and G.D. Mahan, Phys. Rev. B 61, 10651 (2000)

14 A.H. Castro Neto and F. Guinea, Phys. Rev. B 75, 045404 (2007)

15 H. Suzuura and T. Ando, Phys. Rev. B 65, 235412 (2002)

${ }^{16} \mathrm{I}$ am indebted to F. Guinea for suggesting this application and for bringing ref ${ }^{32}$ to my attention.

17 P.R. Wallace, Phys. Rev. 71, 622 (1947)

18 J.C. Slonczewski and P.R. Weiss, Phys. Rev. 109, 272 (1948)
19 J. González, F. Guinea and M.A.H. Vozmediano, Phys. Rev. Lett. 69, 172 (1992)

20 J. González, F. Guinea and M.A.H. Vozmediano, Nucl. Phys. B 406 [FS], 771 (1993)

21 As the space group is symmorphic, the IRs at the $K$ point can be labelled by those of the little point group $C_{3 v}$, which are $A_{1}, A_{2}$ and $E$. Throughout this paper we follow the notation and conventions of ref. ${ }^{2}$

22 J.L. Mañes, F. Guinea and M.A.H. Vozmediano, Phys. Rev. B 75155424 (2007)

23 M. Maeda, Y. Kuramoto and C. Horie, J. Phys. Soc. Jpn. 47337 (1979)

24 A. Sédéki, L.G. Caron and C. Bourbonnais, Phys. Rev. B bf 626975 (2000)

25 J. Maultzsch, S. Reich, C. Thomsen, H. Requardt and P. Ordejón, Phys. Rev. Lett. 92075501 (2004)

26 J. Nilsson, A.H.C. Neto, N.M.R. Peres and F. Guinea, Phys. Rev. B73, 214418 (2006)

27 J. Nilsson, A.H.C. Neto, F. Guinea and N.M.R. Peres, Phys. Rev. Lett. 97, 266801 (2006)

28 K. S. Novoselov, E. McCann, S. V. Morozov, V. I. Falko, M. I. Katsnelson, U. Zeitler, D. Jiang, F. Schedin, and A. K. Geim, Nature Physics 2, 177 (2006)

29 J. C. Meyer, A. K. Geim, M. I. Katsnelson, K. S. Novoselov, T. S. Booth and S. Roth, Nature, March 1, 2007.

30 S.V. Iordanskii and A.E. Koshelev, JETP Lett. 41, 575 (1985)

31 A.F. Morpurgo and F. Guinea, Phys. Rev. Lett. 97, 196804 (2006)

32 S.V. Morozov, K.S. Novoselov, M.I. Katsnelson, F. Schedin, L.A. Ponomarenko, D. Jiang and A.K. Geim, Phys. Rev. Lett. 97, 016801 (2006)

${ }^{33}$ F. Guinea et al. (2007), in progress. 